\title{
Vibration of thin cylindrical shells on an elastic foundation coupled with multiple discrete stiffnesses
}

\author{
Fuchun Yang ${ }^{1,2, a}$ and Wenlei Qiu ${ }^{1}$ \\ ${ }^{1}$ School of Mechanical Engineering, Shandong University, Jinan 250061, China \\ 2 Key Laboratory of High-efficiency and Clean Mechanical Manufacture at Shandong University, Ministry of \\ Education, Jinan 250061, China
}

\begin{abstract}
Vibration properties of thin cylindrical shells on an elastic foundation coupled with multiple discrete stiffnesses were investigated. The discrete stiffnesses were modelled as external forces. Hamilton's principle was applied to deduce the governing equations. To study the natural vibration properties, the wave-like solutions were applied. Then the governing equations were discretized in matrix form to obtain the eigenvalues. The properties of natural frequencies and vibration modes of thin cylindrical shells were studied. The results illustrate that each natural frequency of cylindrical shells is mainly dominated by one vibration mode, that is, flexural, longitudinal and shear modes. The natural frequency will get closer with the increase of circumferential wave numbers. The influences of several parameters to vibration properties of cylindrical shells were also investigated.
\end{abstract}

\section{Introduction}

Vibrations of cylindrical shells have received great attention for over a century in various engineering structures, such as rotor systems of gas turbine engines, high-speed centrifugal separators, rotating satellite structures, and planetary gear sets. This work is motivated by multiple stage planetary gear systems. The ring gears in this structure usually mesh with several planet gears and are supported on elastic foundations.

Many researchers have investigated the vibration of cylindrical shells in different boundary conditions. In the reference [1], the propagation of harmonic waves in thin-walled circular cylindrical shells made of orthotropic and isotropic materials is analysed in the framework of the Sanders linear shell theory. Forsberg [2] studied the influence of general boundary conditions on the modal characteristics of thin cylindrical shells. Weingarten [3] analysed the free vibration of thin cylindrical shells. Warburton [4] studied the natural frequencies and modes for different end conditions. Chung [5] presented a general analytical method for evaluating the free vibration characteristics of a cylindrical shell with classical boundary conditions. Lam and $\mathrm{Wu}$ [6] used the first-order shear deformation theory to presented analytical solutions for thick laminated composite cylindrical shells. $\mathrm{Ng}$ and Lam [7] studied the dynamic stability of simply-supported, isotropic cylindrical panels under combined static and periodic axial forces. In addition, some researchers studied the vibration of rotating cylindrical shells. Kim and Bolton [8] considered the effects of rotation on wave propagation of

\footnotetext{
${ }^{\mathrm{a}}$ Corresponding author: yfc26@163.com
} 
circular cylindrical shells. Omer [9] dealt with the free vibration analysis of rotating laminated cylindrical shells. Padovan [10] obtained the complete set of natural frequencies and corresponding mode shapes for circular cylindrical shells. Huang and Soedel [11] studied the forced response of a special cylindrical shell.

Although the researchers have studied the vibration of cylindrical shells on classical boundary conditions or rotating conditions, there is no study on the vibration of cylindrical shells with multiple discrete stiffnesses as shown in Figure 1. This work will establish the governing equations of thin cylindrical shells on an elastic foundation with multiple discrete stiffnesses and study their vibration properties.

\section{Analytical model}

The vibration model of a cylindrical shell with multiple-stage discrete stiffnesses is shown in Figure1. The fixed coordinate is $\{\mathbf{i}, \mathbf{j}, \mathbf{k}\}$ and the $\left\{\mathbf{e}_{1}, \mathbf{e}_{2}, \mathbf{e}_{3}\right\}$ basis is a local basis on the middle surface of the shell, $u, v$ and $w$ are axial, tangential and radial deformations, respectively. $M$ is a material point on the middle surface and $\theta$ is the relative angle to the basis $\mathbf{i}$, where $0 \leq \theta \leq 2 \pi$. $R$ is the radii of the middle surface. The density of the ring is $\rho$, its elastic modulus is $E$, Poisson's ratio is $\mu$, the length is $L$ and the thickness is $h . k_{i p}$ is discrete stiffness in the $i$ th stage with index $p$. The thin cylindrical shell is supported on the elastic foundation with stiffness $k_{r}$ and $k_{\theta}$ in radial and tangential directions, respectively. The angles between radial directions of the cylindrical shell and each discrete stiffness are $\beta$.

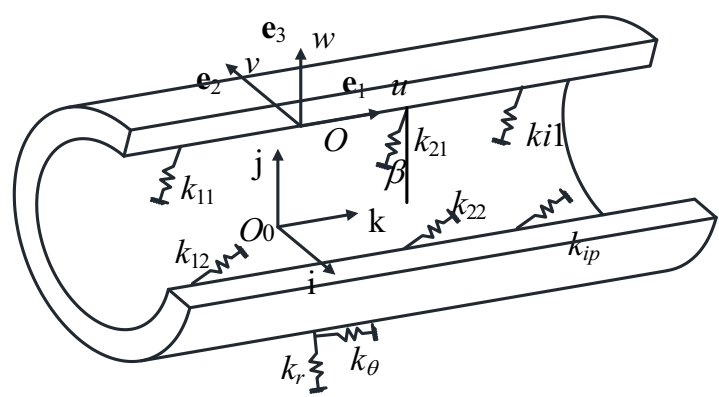

Figure 1. Model of thin cylindrical shell on an elastic foundation coupled with multiple discrete stiffnesses.

By the classical shell theory and the principles of kinetics, the kinetic energy, strain energy of the thin cylindrical shell and the work of external forces caused by discrete stiffnesses were obtained, and the Hamilton's principle was applied to establish the governing equation of thin cylindrical shells as following,

$$
\left\{\begin{array}{l}
\rho h \ddot{u}-\frac{E h}{R^{2}\left(1-\mu^{2}\right)}\left(R^{2} \frac{\partial^{2} u}{\partial z^{2}}+\frac{1-\mu}{2} \frac{\partial^{2} u}{\partial \theta^{2}}+\frac{R(1+\mu)}{2} \frac{\partial^{2} v}{\partial z \partial \theta}+\mu R \frac{\partial w}{\partial z}\right)=0 \\
\rho h \ddot{v}-\frac{E h}{R^{2}\left(1-\mu^{2}\right)}\left[\frac{R(1+\mu)}{2} \frac{\partial^{2} u}{\partial z \partial \theta}+\frac{R^{2}(1-\mu)}{2} \frac{\partial^{2} v}{\partial z^{2}}+\frac{\partial^{2} v}{\partial \theta^{2}}+\frac{\partial w}{\partial \theta}+\frac{h^{2}}{12}\left(\frac{1-\mu}{2} \frac{\partial^{2} v}{\partial z^{2}}+\frac{\partial^{2} v}{R^{2} \partial \theta^{2}}-\frac{\partial^{3} w}{\partial z^{2} \partial \theta}\right.\right. \\
\left.\left.\quad-\frac{\partial^{3} w}{R^{2} \partial \theta^{3}}\right)\right]+k_{\theta} v+\sum_{i}^{M_{n}} \sum_{p}^{N_{s}} k_{i p}\left(v\left(z_{m i}, \theta_{i p}, t\right) \sin ^{2} \beta+w\left(z_{m i}, \theta_{i p}, t\right) \sin \beta \cos \beta\right) \delta\left(z-z_{m i}\right) \delta\left(\theta-\theta_{i p}\right)=0 \\
\rho h \ddot{w}+\frac{E h}{R^{2}\left(1-\mu^{2}\right)}\left[\mu R \frac{\partial u}{\partial z}+\frac{\partial v}{\partial \theta}+w+\frac{h^{2}}{12}\left(R^{2} \frac{\partial^{4} w}{\partial z^{4}}+2 \frac{\partial^{4} w}{\partial z^{2} \partial \theta^{2}}+\frac{\partial^{4} w}{R^{2} \partial \theta^{4}}-\frac{\partial^{3} v}{\partial z^{2} \partial \theta}-\frac{\partial^{3} v}{R^{2} \partial \theta^{3}}\right)\right]+k_{r} w \\
\quad+\sum_{i}^{M_{n}} \sum_{p}^{N_{s}} k_{i p}\left(v\left(z_{m i}, \theta_{i p}, t\right) \sin \beta \cos \beta+w\left(z_{m i}, \theta_{i p}, t\right) \cos ^{2} \beta\right) \delta\left(z-z_{m i}\right) \delta\left(\theta-\theta_{i p}\right)=0
\end{array}\right.
$$


where $\delta$ is Dirac delta function, $i$ is the group index of discrete stiffnesses on the same circular, $p$ is the index number in a group, $M_{n}$ and $N_{s}$ are the number of group and the number of discrete stiffnesses in one group, respectively. $z_{m i}$ and $\theta_{i p}$ are the positions of discrete stiffnesses in the axial direction and circumferential direction, respectively. To investigate the vibration properties of thin cylindrical shells, the set of displacements were assumed to have the wave-like forms,

$$
\begin{aligned}
& u_{m n}(z, \theta, t)=A_{m n} \cos \frac{m \pi z}{L} e^{\left(j \omega_{m n} t-j n \theta\right)} \\
& v_{m n}(z, \theta, t)=j B_{m n} \sin \frac{m \pi z}{L} e^{\left(j \omega_{m n} t-j n \theta\right)} \\
& w_{m n}(z, \theta, t)=C_{m n} \sin \frac{m \pi z}{L} e^{\left(j \omega_{m n} t-j n \theta\right)}
\end{aligned}
$$

where $m$ and $n$ represent the axial half wave number and circumferential wave number, respectively. Equation (4)-(6) were substituted into Equation (1)-(3) and explored the orthogonality properties of $\cos m \pi z / L, \sin m \pi z / L, e^{j n \theta}$ to yield the eigenvalue equations. The equations were written in matrix form as

$$
\mathbf{M}_{m n} \ddot{\mathbf{x}}_{m n}+\left(\mathbf{K}_{m n}+\mathbf{K}_{m n}^{b}+\mathbf{K}_{m n}^{m}\right) \mathbf{x}_{m n}=\mathbf{0}
$$

where $\mathbf{x}=\left[A_{m n}, B_{m n}, C_{m n}\right]$ and $\mathbf{M}_{m n}$ is mass matrix, $\mathbf{K}_{m n}, \mathbf{K}_{m n}^{b}, \mathbf{K}_{m n}^{m}$ are corresponding system stiffnesses, foundation stiffnesses and discrete stiffness matrix, respectively. Equation (7) is an equation about the variables $A_{m n}, B_{m n}, C_{m n}$, and the eigenvalue problem can be solved by standard eigenvalue method.

\section{Numerical results}

Since this study is motivated by multiple stage planetary gear systems, the parameters of a thin cylindrical shell which denotes a ring gear in the planetary gear system are given as $\rho=7800 \mathrm{~kg} / \mathrm{m}^{3}$, $E=206 \mathrm{GPa}, \mu=0.3, k_{r}=k_{\theta}=1 \times 10^{8} \mathrm{~N} / \mathrm{m}, k_{i p}=5 \times 10^{8} \mathrm{~N} / \mathrm{m}, R=160 \mathrm{~mm}, h=10 \mathrm{~mm}, L=300 \mathrm{~mm}, \beta=1.22, z_{m i}=L / 6$, $L / 2,5 L / 6, \theta_{i p}=0,2 \pi / 3,4 \pi / 3, i=1,2,3, p=1,2,3, M_{n}=N_{s}=3$, the discrete stiffnesses are axially and circumferentially equispaced and the phases between adjacent axial group of the discrete stiffnesses are set to $\pi / 4$.

The eigenvalue equation obtained from Equation (7) is six order equation, that is, there are six natural frequencies associated with the $(m, n)$ th wave-like solution. However, the negative and positive solutions appear as pair with the same magnitude but opposite sign. Thus, for each positive or negative $n$, the three positive natural frequencies are chosen and the negative natural frequencies are discarded. There are then only three distinct natural frequencies associated with each $(m, n)$ wave mode, whether $n>0$ or $n<0$. And each frequency is primarily associated with a particular wave type, that is, shear, or flexural, longitudinal. In what follows, we present the results of numerical investigations, which were carried out by a range of $m$ from 1 to 5 and $n$ from 1 to 10 .

Figure 2a illustrates the natural frequency of the thin cylindrical shells on an elastic foundation with multiple group of discrete stiffnesses. It can be seen that the natural frequencies will increase with the increasing of $m$ or $n$ and the natural frequencies can be classified into three groups. And the three groups of frequencies correspond to flexural, longitudinal and shear modes, respectively. For each $(m, n)$ th order, the vibration of the cylindrical shell is comprised of three vibration modes, but one of the three modes is dominant and the other two modes are ignorable. By analysing the vibration mode corresponding to each frequency in Figure $2 \mathrm{a}$, it can be found that the lower frequencies mainly associate with flexural mode as shown in Figure $3 \mathrm{a}$, the intermediate frequencies mainly associate with longitudinal mode as shown in Figure 3b, and the higher frequencies mainly associate with shear mode as shown in Figure 3c. Figure $2 b$ shows the natural frequencies of shear mode with axial half wave number $m$ equals from 1 to 5 and circumferential wave number $n$ equals from 1 to 10 . It can be 
observed that the natural frequencies of the cylindrical shell increase with $n$ at each $m$ nonlinearly and the natural frequencies get closer with the increase of $n$ and $m$.

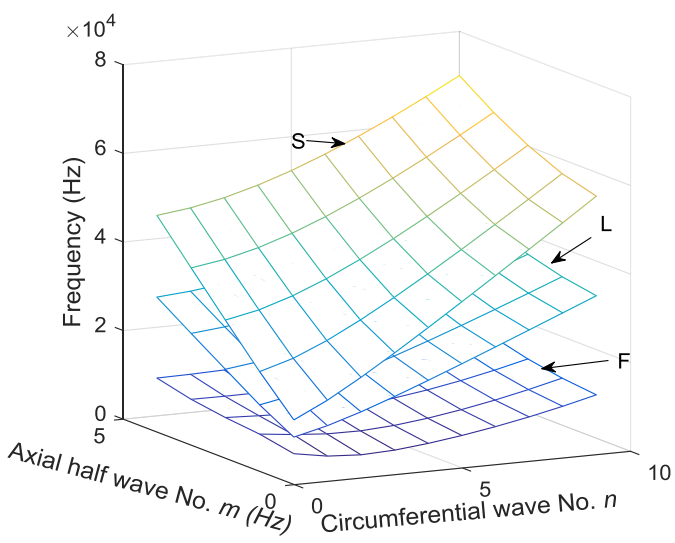

(a)

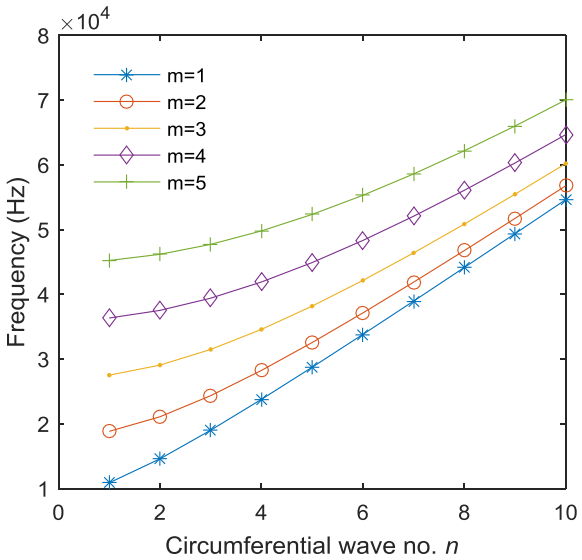

(b)

Figure 2. (a) Natural frequencies of thin cylindrical shell with $m=1 \sim 5$ and $n=1 \sim 10$; (b) Natural frequencies of shear mode.
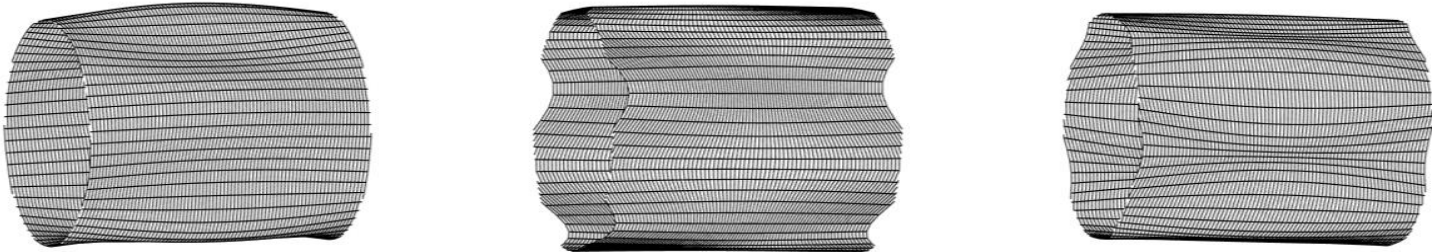

Figure 3. Vibration modes (a) flexural mode; (b) longitudinal mode; (c) shear mode.

The influences of discrete stiffnesses and support stiffnesses to the natural frequencies are illustrated in Figure 4. It can be seen from Figure 4 that with the increase of two types of stiffnesses the natural frequencies of the three modes will all increase. However, Figure 4a shows that the discrete stiffnesses mainly affect the natural frequencies of shear mode, i.e., the high order natural frequencies, and the natural frequencies of the other two modes change very slightly. Figure $4 \mathrm{~b}$ shows that the support stiffnesses mainly affect the natural frequencies of flexural mode, i.e., the low order natural frequencies. And the intermediate natural frequencies of longitudinal mode almost have no change with the increase of support stiffness.

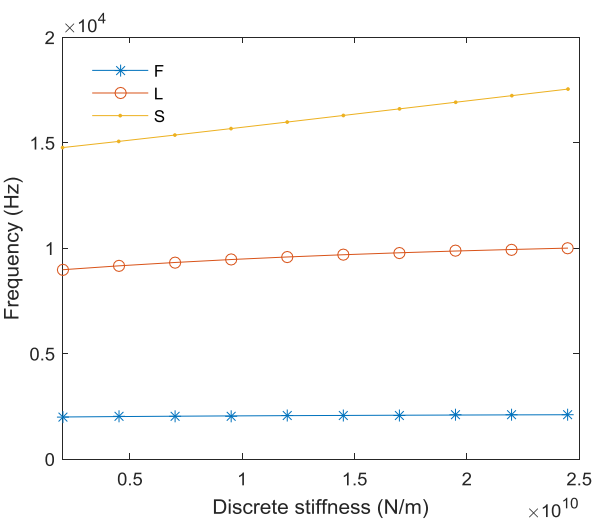

(a)

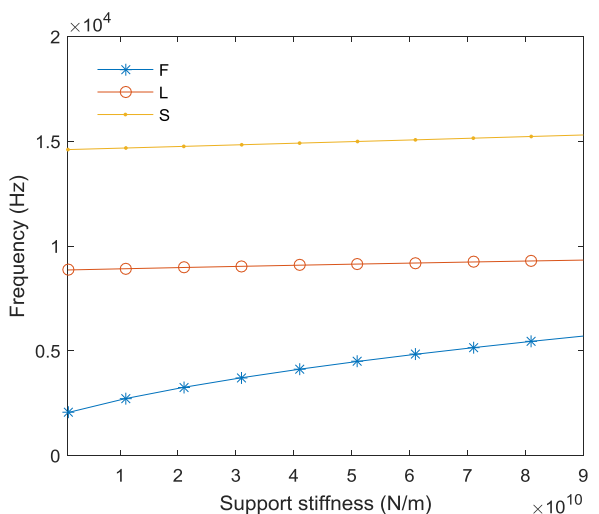

(b)

Figure 4. Natural frequencies of cylindrical shell vs. two types of stiffnesses (a) discrete stiffness; (b) support stiffness where $m=1$ and $n=2$. 


\section{Conclusions}

Vibration properties of thin cylindrical shells with multiple-stage discrete stiffnesses and foundation stiffnesses were investigated in this paper. The governing equations were obtained by the Hamilton's principle. In order to get the uniform equations, the discrete stiffnesses were modelled as external forces. The wave-like solutions were applied to study the natural vibration properties and the governing equations were discretized in matrix form to obtain the eigenvalues. The properties of natural frequencies and vibration modes of cylindrical shells were studied. The influences of discrete stiffnesses and foundation stiffnesses to natural frequencies of thin cylindrical shells were also investigated. The results show that each natural frequency is mainly dominated by one vibration mode, that is, flexural, longitudinal and shear modes. The natural frequency will get closer with the increase of circumferential wave numbers. The discrete stiffnesses mainly affect the higher order natural frequencies and the support stiffnesses mainly affect the lower order natural frequencies.

\section{Acknowledgements}

This work was supported by the National Natural Science Foundation of China (Grant numbers 51775309) and Young Scholars Program of Shandong University.

\section{References}

1. T. Chen, C. Bert. Nucl. Eng. Des., 42 (1977)

2. K. Forsberg. AIAA J 2 (1964)

3. V. Weingarten. Aero. Corp. Sys. R\&P Div., El Segundo, California, Report No. 169 (1962)

4. G. Warburton. J. Mech. Eng. Sci., 7 (1965)

5. Chung H. J. Sound Vib., 74 (1981)

6. K. Lam, Q. Wu. J. Compo., 31 (2000)

7. T. Ng, K. Lam, J. Reddy. Intl J. Solids Struct., 36 (1999)

8. Y. Kim, J. Bolton. J. Sound Vib., 275 (2004)

9. C. Omer. Thin Wall. Struct., 25 (2007).

10. J. Padovan. J. Sound Vib., 31 (1973)

11. S. Huang, W. Soedel. J. Sound Vib., 118 (1987) 